\title{
Evaluation of Turbulence Models for the Design of Continuously Adapted Riblets
}

\author{
Karsten Oehlert, Christoph A. Million and Konrad Hartung \\ Jade University of Applied Sciences, Wilhelmshaven 26389, Germany
}

\begin{abstract}
The extensive investigations during the last decades have verified the potential of ideal shaped riblet geometries for viscous drag reduction in the turbulent boundary layer. Basic requirement is an adaption of the riblet dimensions in terms of the local flow conditions. However, due to the complex manufacturing process of micro-scale surface structures most experimental measurements were conducted using riblets with constant dimensions. Therefore, the drag reducing potential of riblets could not be exploited. Thanks to the rapid development in micro structuring technologies it is now possible to manufacture continuously adapted riblets in almost industrial processing scales. To determine an appropriated area for the riblet application and continuously adapted riblet dimensions for the National Renewable Energy Laboratory (NREL) airfoil S809 information on the location of the laminar-turbulent transition and the wall shear stress distribution are required. For this purpose, numerical simulations were conducted using the transitional $\mathrm{kk}_{\mathrm{L}}-\omega$ and $\gamma-\mathrm{Re}_{\Theta}$ turbulence models as well as the fully turbulent SST implementation. Both transitional models show a sufficient precise prediction of the transition onset location in comparison with experimental data. Depending on the applied turbulence model computed riblet dimensions exhibit a certain deviation whereby a significant effect on the drag reduction can be expected.
\end{abstract}

Key words: Riblets, drag reduction, transition modeling, wind turbines, laser structuring.

\section{Nomenclature}

\section{$c \quad$ airfoil chord length, $\mathrm{m}$}

$h \quad$ riblet height, $\mathrm{m}$

$h^{+} \quad$ dimensionless riblet height

$k \quad$ turbulent kinetic energy, $\mathrm{J} / \mathrm{kg}$

$k_{L} \quad$ laminar kinetic energy, $\mathrm{J} / \mathrm{kg}$

$P \quad$ pressure, $\mathrm{Pa}$

$s \quad$ riblet spacing, $\mathrm{m}$

$s^{+} \quad$ dimensionless riblet spacing

$t \quad$ riblet tip width, $\mathrm{m}$

$t^{+} \quad$ dimensionless riblet tip width

$u_{\tau} \quad$ shear velocity, $\mathrm{J} /(\mathrm{kg} \mathrm{K})$

$x_{t} \quad$ transition onset location, $\mathrm{m}$

$y \quad$ dimensionless wall spacing

\section{Greek Symbols}

$\begin{array}{ll}a & \text { angle of attack } \\ \gamma & \text { intermittency } \\ o & \text { eddy dissipation, } \mathrm{J} /(\mathrm{kg} \mathrm{s}) \\ \mu & \text { dynamic viscosity, } \mathrm{kg} /(\mathrm{m} \mathrm{s}) \\ \mu \tau & \text { eddy viscosity }\end{array}$

Corresponding author: Karsten Oehlert, Dr.-Ing., professor, research fields: drag reduction and riblets.

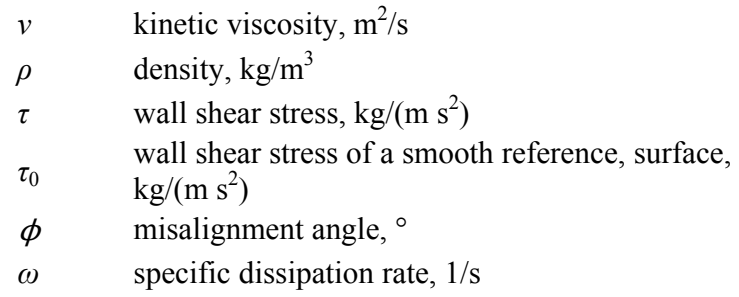

\section{Introduction}

Modern lifestyles and growth of humanity demand a steady supply of energy, which nowadays is mainly provided by fossil resources. Their limitation and the increasing global warming have led to a rethinking in the area of energy production. The research focus has shifted within the last two decades from fossil energy production to the development and improvement of renewable energy technologies. To increase the efficiency of wind energy production, which is one of the most promising renewable energy technologies, occurring losses, such as tip and profile losses must be reduced. For instance, in technical operation of wind turbines the pressure gradient induced by-pass flow at 
the tip of the rotor blade can be reduced through the application of winglets such as used on modern aircrafts. Profile losses occur in most fluid mechanical applications, for instance on wind turbine airfoils, aircrafts or turbomachinery applications, and are caused by the viscous drag in the boundary layer. To reduce the viscous drag mainly two approaches have been investigated focusing on delaying the location of the laminar-turbulent transition and modifying the turbulent structure within the boundary layer. Since the 1970's there have been extensive investigations on the viscous drag reduction by the application of periodically arranged microscopic surface structures, so-called riblets, inspired by the drag reducing mechanisms of shark skin. Different riblet configurations have been investigated among others e.g. by Bechert et al. [1] showing that stream-wise oriented riblets provide the potential to reduce the wall shear stress up to $10 \%$ in reference to a smooth surface. Basic requirement to exploit the drag reducing effect of riblets is to adjust the riblet dimensions continuously to the local flow conditions. However the manufacturing process of these microscale structures by grinding, milling, coating, varnishing, rolling, and laser based processes in industrial relevant scales and with the required quality has been so far an unsurmountable challenge [2]. Therefore, most of the experimental investigations have been performed using vinyl adhesive backed riblet sheets with constant dimensions, such as provided e.g. by the 3M Corp (e.g. by Oehlert [3]), which restricts the possible drag reduction. Due to the recent developments in high-rate laser micro structuring processing continuously adapted riblets can now be manufactured in almost industrial relevant scales. To provide the information required to determine an appropriate area for the riblet application and continuously adapted riblet dimensions on the National Renewable Energy Laboratory (NREL) airfoil S809 numerical studies were conducted using the transitional $\mathrm{kk}_{\mathrm{L}}-\omega$ and $\gamma-\mathrm{Re}_{\Theta}$ turbulence models as well as the fully turbulent SST implementation. The major goal of this paper is to determine the most suitable turbulence model for the design process.

\section{General Information}

\subsection{Turbulent Shear Stress Reduction}

Fig. 1a shows the primary stripe-like turbulent structures in the viscous sublayer of an turbulent boundary layer, which consists of an alternating array of low-speed and high-speed streaks [4]. These superimposed coherent vortices support the momentum exchange in the near wall region. Therefore, in comparison to a laminar boundary layer fluid with higher momentum interacts with the surface leading to an overall increased wall shear stress, which results in an increased viscous drag. A reduction of the viscous drag in turbulent flows can be achieved by decreasing
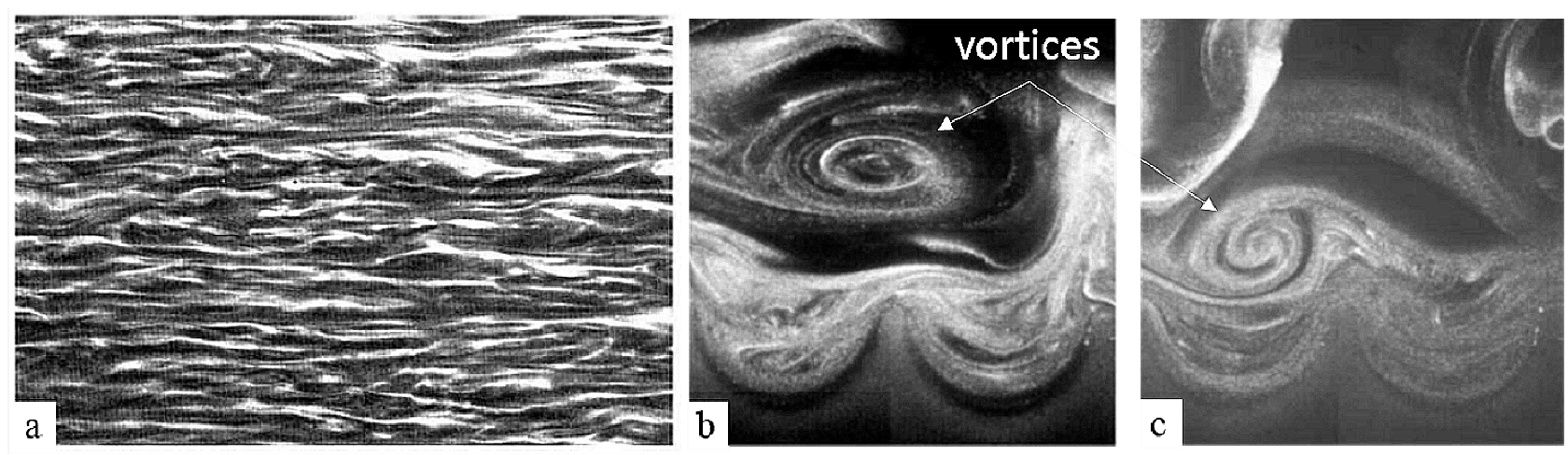

Fig. 1 Flow visualization of stream-wise vortices: (a) top view of a viscous sublayer in the turbulent boundary layer (main flow from left to right) [5], vertical cross-section of riblets with semi-circular grooves; (b) drag reducing configuration [6]; (c) drag increasing configuration [6]. 
the momentum exchange in the boundary layer. S.-J. Lee and S.-H. Lee, [6] visualized the impact of riblets on the near wall flow using a synchronized smoke-wire technique over a smooth and a riblet structured surfaces (Figs. $1 \mathrm{~b}$ and 1c). According to Bechert et al. [4] riblets hamper the lateral components of the vortices and additionally elevate the dominant vortex cores. The direct numerical simulations (DNS) of the boundary layer flow conducted by Goldstein et al. [7], as well as experimental studies of Vukoslavčević et al. [8], provided evidence for the drag reducing mechanism of riblets. In case of a correct dimensioning of the riblet spacing the dominant vortices only interact with the riblet tips. Therefore the overall wetted surface in contact with fluid of high momentum is decreased compared to a smooth surface, leading to a significant viscous drag reduction (Fig. 1b). In case of an oversized riblet spacing (Fig. 1c) on the other hand the vortices increasingly occur within the riblet valleys (Fig. 1b). As a result an overall enlarged surface interacts with fluid of high momentum resulting in higher viscous drag [1]. Summarizing it can be noted that riblets develop their drag reducing potential exclusively in the turbulent boundary layer by decreasing the momentum exchange in the viscous sublayer. An application of riblets within the laminar boundary layer should be avoided as it would have a comparable effect as the presence of surface roughness increasing the viscous drag. Information on the location of the laminar-turbulent transition is therefore necessary to determine an appropriated area for the riblet application.

\subsection{Riblet Geometries and Configuration}

Bechert et al. [1] and Walsh [9] carried out extensive research regarding the wall shear stress reducing effect of different riblet configurations and geometries with sawtooth, trapezoid and blade shaped cross-sections. Therefore the riblet spacing $s$ and the riblet height $h$, the riblet tip width $t$, the opening angle $\alpha$ and the misalignment angle $\phi$, which describes the angle between the main flow direction and the riblet orientation, are used for the riblet characterization. For a better comparison of experimental result on different riblet configurations and flow conditions dimensionless parameters, measured in wall units and labelled with an additional ${ }^{+}$-symbol as shown in Eq. (1) are introduced. Here $\tau_{0}$ refers to the wall shear stress of a smooth reference surface, $u_{\tau}$ is the shear velocity; $\rho$ and $v$ are the density and the kinematic viscosity respectively.

$$
\begin{gathered}
s^{+}=s\left(\frac{u_{\tau}}{v}\right) ; h^{+}=h\left(\frac{u_{\tau}}{v}\right) ; t^{+}=t\left(\frac{u_{\tau}}{v}\right) \\
u_{\tau}=\sqrt{\frac{\tau_{0}}{\rho}}
\end{gathered}
$$

Based on various investigations, Walsh [9] concluded that for riblets with a sawtooth cross-section and a height to spacing ratio of $h / s=1$ a maximum wall shear stress reduction in reference to a smooth surface in range of $\Delta \tau / \tau_{0}=-7.8 \%$ can be achieved. In the scope of investigations on different riblet geometries in an oil channel, Bruse [10] found that the wall shear stress reducing effect of riblets could further be increased by the use of riblet with trapezoid and band-shaped cross-sections. For blade shaped riblets with a dimensionless riblet spacing of $s^{+}$ $=17$, a height of $h=0.5 \mathrm{~s}$ and a tip width of $t=0.002$ $\mathrm{s}$ the overall highest wall shear stress reduction in range of up to $\Delta \tau / \tau_{0}=-9.9 \%$ [10] was measured (Fig. 2). However due to the low mechanical durability of blade shaped riblets, riblets with a trapezoid cross-section are preferred for technical application. For this kind of cross-section a maximum wall shear stress reduction of $\Delta \tau / \tau_{0}=-8.2 \%$ was measured for a height to spacing ratio of $h / s=0.5$ [10] and a dimensionless riblet spacing of $s^{+}=17$.

\section{Numerical Method}

As reported in Section 2.1, the design of continuously adapted riblets requires information on 


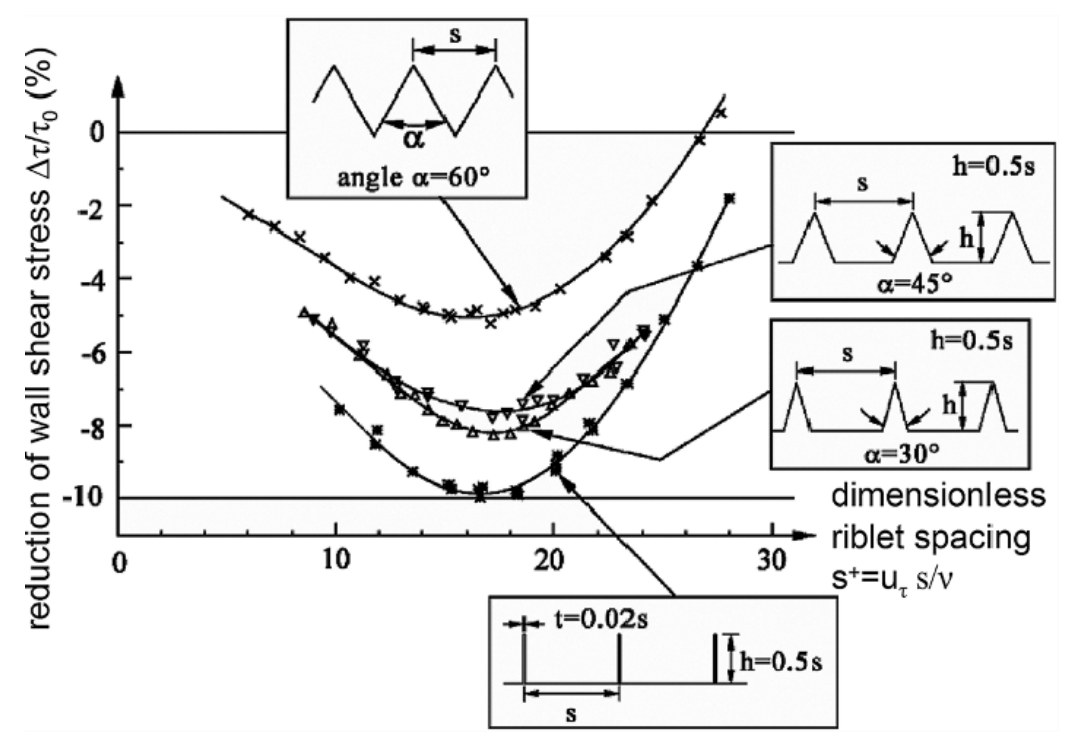

Fig. 2 Wall shear stress reduction for different riblet configurations [1].

the location of the laminar-turbulent transition and according to Eqs. (1) and (2) additional information on the local wall shear stress distribution. Therefore, steady state 2D incompressible flow simulations over the S809 airfoil were performed in ANSYS Fluent using the transitional $\mathrm{kk}_{\mathrm{L}}-\omega$ and $\gamma-\mathrm{Re}_{\Theta}$ turbulence models. The evaluations of the turbulence models are conducted in terms of a precise prediction of the transition onset location and a reliable wall shear stress distribution. Numerical results on the predicted transition onset location are compared to the experimental investigations of low turbulence wind tunnel measurements at Delft University of Technology reported by Somers [11]. Due to unavailable literature data on the wall shear stress distribution on the $\mathrm{S} 809$ airfoil the numerical results based on the $\mathrm{kk}_{\mathrm{L}}-\omega$ and $\gamma-\mathrm{Re}_{\Theta}$ models are compared to the results of the fully turbulent SST implementation.

\subsection{Turbulence Modelling}

In most engineering and aerospace applications transitional boundary layer flow occurs. To avoid the computational cost of resolving the turbulent and transitional phenomena numerically the Reynolds-averaged Navier-Stokes equations (RANS) are solved, which average the Navier-Stokes equations over decomposed mean and fluctuation components. For an incompressible Newtonian fluid the continuity and momentum equation can be written as

$$
\begin{gathered}
\frac{\partial(\rho)}{\partial t}+\frac{\partial\left(\rho \bar{u}_{i}\right)}{\partial x_{i}}=0 \\
\frac{\partial\left(\rho \bar{u}_{i}\right)}{\partial t}+\frac{\partial\left(\rho \bar{u}_{i} \bar{u}_{j}\right)}{\partial x_{i}}= \\
-\frac{\partial \bar{p}}{\partial x_{i}}-\frac{\partial \bar{\tau}_{i j}}{\partial x_{j}}-\frac{\partial\left(\rho \overline{u_{i}^{\prime} u_{j}^{\prime}}\right)}{\partial x_{i}}
\end{gathered}
$$

where the additional Reynolds stress term $\left(\rho \overline{u_{i}^{\prime} u_{j}^{\prime}}\right)$ needs to be modelled.

\subsubsection{SST}

For industrial applications the eddy-viscosity based $k-\omega$ and $k-\varepsilon$ model, which solve the two additional transport equations for the turbulent kinetic energy $k$ and the specific dissipation rate $\omega$ or the turbulent eddy dissipation rate $\varepsilon$ respectively have established themselves. The two-equation Shear Stress Transport Model (SST) developed by Menter [12] effectively blends the robust and accurate formulation of the turbulence/frequency-based $k$ - $\omega$ model in the near-wall region with the free-stream independence of the turbulence/dissipation-based $k-\varepsilon$ model to achieve an optimal formulation for a wide range of 
applications. Therefore, the standard $k$ - $\varepsilon$ model is transformed into $k$ - $\omega$ denotation by substituting $\varepsilon=k \omega$ [13]. The transport equation for the turbulent kinetic energy and the specific dissipation rate read

$$
\begin{gathered}
\frac{\partial(\rho k)}{\partial t}+\frac{\partial\left(\rho k u_{i}\right)}{\partial x_{i}}=\frac{\partial}{\partial x_{j}}\left[\left(\mu+\frac{\mu_{k}}{\sigma_{k}}\right) \frac{\partial k}{\partial x_{j}}\right] \\
+P_{k}-Y_{k}+S_{k^{\prime}} \\
\frac{\partial(\rho \omega)}{\partial t}+\frac{\partial\left(\rho \omega u_{i}\right)}{\partial x_{i}}=\frac{\partial}{\partial x_{j}}\left[\left(\mu+\frac{\mu_{k}}{\sigma_{k}}\right) \frac{\partial \omega}{\partial x_{j}}\right] \\
+P_{\omega}-Y_{\omega}+S_{\omega}+D_{\omega^{\prime}}
\end{gathered}
$$

where $P_{\omega}$ and $P_{k}$ represent the production terms. The dissipation and the source terms are given by $Y_{\omega}$ and $Y_{k}$ and $S_{k}$ and $S_{\omega}$ respectively. The term $D_{\omega}$ represents a blending function which ensures that the $k-\omega$ model is activated in the near wall region and the $k-\varepsilon$ model in free shear flow [13]. The performance of the SST model has been studied in a large number of cases showing an overall higher accuracy on capturing the onset of separation under pressure gradients. However, the model is not capable of modelling the laminar turbulent transition in the boundary layer and thus on its own insufficient to determine the optimal area for the riblet application.

\subsection{2 $\gamma-\operatorname{Re}_{\Theta}$}

The four-equation transition SST model also known as the $\gamma-\operatorname{Re}_{\Theta}$ model is a local formulation based on transition model in which only local flow variables, gradients, and the wall distance are used to trigger the transition in the boundary layer. In addition to the standard SST model two further transport equations for the intermittency $\gamma$ and the momentum thickness Reynolds number $\overline{R e_{\theta}}$ are solved. The transport equation can be written as

$$
\begin{gathered}
\frac{\partial(\rho \gamma)}{\partial t}+\frac{\partial\left(\rho \gamma u_{j}\right)}{\partial x_{j}}=\frac{\partial}{\partial x_{j}}\left[\left(\mu+\frac{\mu_{t}}{\sigma_{f}}\right) \frac{\partial \gamma}{\partial x_{j}}\right] \\
+P_{\gamma}-E_{\gamma^{\prime}} \\
\frac{\partial\left(\rho \overline{R e}_{\theta}\right)}{\partial t}+\frac{\partial\left(\rho \overline{R e}_{\theta} u_{j}\right)}{\partial x_{j}}= \\
\frac{\partial}{\partial x_{j}}\left[\sigma_{\theta}\left(\mu+\mu_{t}\right) \frac{\partial \overline{R e}_{\theta}}{\partial x_{j}}\right]+P_{\theta}
\end{gathered}
$$

where $P_{\gamma}$ and $P_{\Theta}$ are the transition and $E_{\gamma}$ is the relaminarization source term. The coupling of the transition model with the standard SST model is performed by modifying the $k$ - $\omega$ equation with the effective intermittency $\gamma_{\text {eff }}$ in Eqs. (9)-(11).

$$
\begin{gathered}
\frac{\partial(\rho k)}{\partial t}+\frac{\partial\left(\rho k u_{i}\right)}{\partial x_{i}}=\frac{\partial}{\partial x_{j}}\left[\left(\mu+\frac{\mu_{k}}{\sigma_{k}}\right) \frac{\partial k}{\partial x_{j}}\right] \\
+P_{\dot{k}}^{*}-Y_{\dot{k}}^{*}+S_{k^{\prime}} \\
P_{\dot{k}}^{*}=\gamma_{e f f} P_{k^{\prime}} \\
Y_{\dot{k}}^{*}=\min \left(\max \left(\gamma_{e f f}, 0.1\right), 1.0\right) Y_{k}
\end{gathered}
$$

The transport equation for the intermittency is used to trigger the transition locally by turning on the production term of the turbulent kinetic energy in the modified $k-\omega$ equation downstream of the transition onset. The transport equation for the momentum thickness Reynolds number is necessary to capture the nonlocal influence of the turbulent intensity and to impose the transition onset criteria estimated by an empirical correlation to the intermittency equation [14].

\section{$3.2 k k_{L^{-}}-\omega$}

The third turbulence model conducted within this study is the three-equation $k k_{L}-\omega$ model initially proposed by Walters and Leylek [15]. The model is based on the assumption that velocity fluctuation in the pre-transitional region can be divided into small-scale vortices contributing to turbulence production and large-scale longitudinal vortices contributing to the production of non-turbulent fluctuation. The transition process is modelled by the effect of energy transfer from the laminar kinetic energy of large-scale longitudinal vortices to the turbulent kinetic energy of small-scale vortices with a concurrent reduction in turbulent length scale [15]. The implementation is based on the $k$ - $\omega$ framework and solves a third transport equation for the laminar kinetic energy $k_{L}$ to predict the magnitude of low frequency velocity fluctuations that have been identified triggering transition in the boundary layer. The transport equations for the turbulent and laminar kinetic energy and the specific dissipation rate are 
given by Eqs. (12)-(14).

$$
\begin{gathered}
\frac{\partial(\rho k)}{\partial t}+\frac{\partial\left(\rho k u_{i}\right)}{\partial x_{i}}=\frac{\partial}{\partial x_{j}} \\
{\left[\left(\mu+\frac{\mu_{t}}{\sigma_{k}}\right) \frac{\partial k_{T}}{\partial x_{j}}\right]-P_{k}+R_{B P}} \\
+R_{N A T}-D_{T}+\omega k_{T^{\prime}}, \\
\frac{\partial\left(\rho k_{L}\right)}{\partial t}+\frac{\partial\left(\rho k_{L} u_{i}\right)}{\partial x_{i}}=\frac{\partial}{\partial x_{j}}\left[\mu \frac{\partial k_{L}}{\partial x_{j}}\right] \\
-P_{k L}+R_{B P}+R_{N A T}-D_{L^{\prime}} \\
\frac{\partial(\rho \omega)}{\partial t}+\frac{\partial\left(\rho \omega u_{i}\right)}{\partial x_{i}}=\frac{\partial}{\partial x_{j}}\left[\left(\mu+\frac{\mu_{\omega}}{\sigma_{\omega}}\right) \frac{\partial \omega}{\partial x_{j}}\right] \\
C_{\omega 1} \frac{\omega}{k_{T}} P_{k T}+\left(\frac{C_{\omega R}}{f_{\omega}}-1\right) \frac{\omega}{k_{T}} \\
\left(R_{B P}-R_{N}\right)-C_{\omega 2} \omega^{2} \\
+C_{\omega 3} f_{\omega} \alpha_{T} f_{\omega}^{2} \frac{\sqrt{k_{T}}}{d^{3}}
\end{gathered}
$$

Here, the terms $R_{B P}$ and $R_{N A T}$ appearing with opposite signs in the transport equations for laminar and turbulent kinetic energy are related to the transition mechanism for bypass and natural transition, respectively. The terms $D_{T}$ and $D_{L}$ represent the non-isotropic part of the near wall dissipation of the turbulent and laminar kinetic energy. In the transport equation for the specific dissipation rate, the second term on the right hand side is the transition production term intended to reduce turbulent length scales during the transition process [15]. The fourth term on the right hand side decreases the length scale in the outer region of the turbulent boundary layer necessary to ensure an adequate predication of the wake region [16].

\subsection{Airfoil and Numerical Setup}

For this study, the NREL airfoil S809, which is a 21\% thick airfoil especially designed for the application of horizontal axis wind turbines (HAWT) is used. The S809 airfoil was developed using the Eppler design code [17]. Thus, a table of coordinates defines the geometry of the airfoil. To provide the high surface resolution necessary for numerical simulations spline interpolation is used. The computational domain consists of a semi-circular velocity inlet with a radius of 10 chord lengths. The pressure outlet is set to be at 20 chord lengths downstream measured from the trailing edge of the airfoil. Furthermore, the upper and lower boundaries of the computational domain are defined as velocity inlet and pressure outlet respectively. The Reynolds-averaged Navier-Stokes equations are solved using a pressure based finite-volume approach and a second-order upwind spatial discretization on a structured CH-type body-fitted mesh. For the velocity pressure coupling the semi-implicit method for pressure-linked equations (SIMPLE) was employed. The inlet turbulence intensity and the turbulent viscosity ratio were set to $0.2 \%$ and 10 , respectively. Simulations were conducted for the angles of attack $\alpha$ $=0^{\circ}, 4.1^{\circ}, 5.13^{\circ}, 6.16^{\circ}, 8.2^{\circ}, 9.22^{\circ}, 11.22^{\circ}, 16.2^{\circ}$ and the Reynolds number $R e=2 \times 10^{6}$ by adjusting the velocity components at the inlet. To ensure the numerical results are independent of the grid size, the number of cells was increased until negligible differences of the non-dimensional forces were attained. Starting point for the grid independence study was a coarse grid with a total number of 44,500 cells. The number of cells was increased to 176,115 cells and 708,225 cells for the medium and fine grid respectively. For all grids, the mesh density in the boundary layer was chosen such that the requirement of $y^{+}<1$ for wall resolved turbulence modeling is satisfied. As shown in Figs. 3 and 4, for an angle of attack of $\alpha=0^{\circ}$ and $\alpha=8.2^{\circ}$ the differences for the aerodynamic lift and drag coefficient become negligible for the medium grid. In comparison to the fine grid the computed lift and drag coefficients differ by less than $1 \%$. Therefore, a further increase of the mesh resolution would lead to a disproportionate change of the aerodynamic coefficient in relation to the computational time necessary. Thus, the medium grid illustrated in Figs. 5 and 6 was applied for further investigations. 


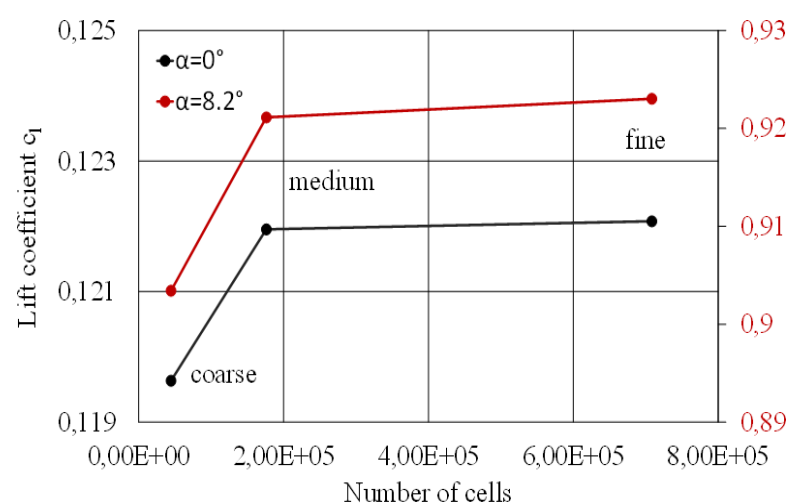

Fig. 3 Lift coefficient for different grid sizes for $\alpha=0^{\circ}$ and $\alpha=8.2^{\circ}$.

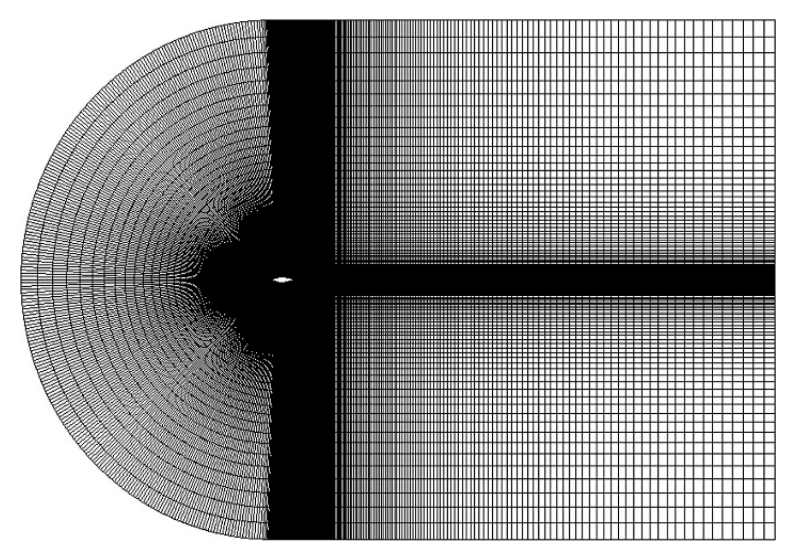

Fig. 5 Structure of the CH-type mesh for the S809 airfoil.

\section{Results and Discussion}

In literature usually the normalized position of the transition onset location is used to compare experimental and numerical results. Therefore, the evaluation of the turbulence models in terms of being capable of determining an appropriated area for the riblet application is conducted based on the precise prediction of the transition onset location. The onset of the fully developed turbulent boundary layer appropriated for the riblet application is subsequent determined based on the wall shear stress distribution. According to the definitions of the turbulence models the transition onset location was determined by an initially increasing turbulent kinetic energy in the near wall region (Fig. 7). Figs. 8 and 9 give an overview over the predicted transition onset locations for the $k k_{L}-\omega$ and $\gamma-\operatorname{Re}_{\Theta}$ model in comparison to the experimental data reported by Somers [11]. Both

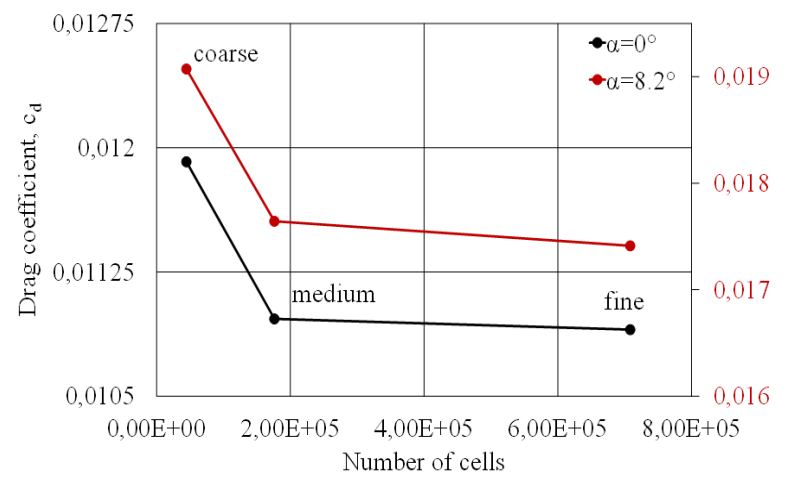

Fig. 4 Drag coefficient for different grid sizes for $\alpha=0^{\circ}$ and $\alpha=8.2^{\circ}$.

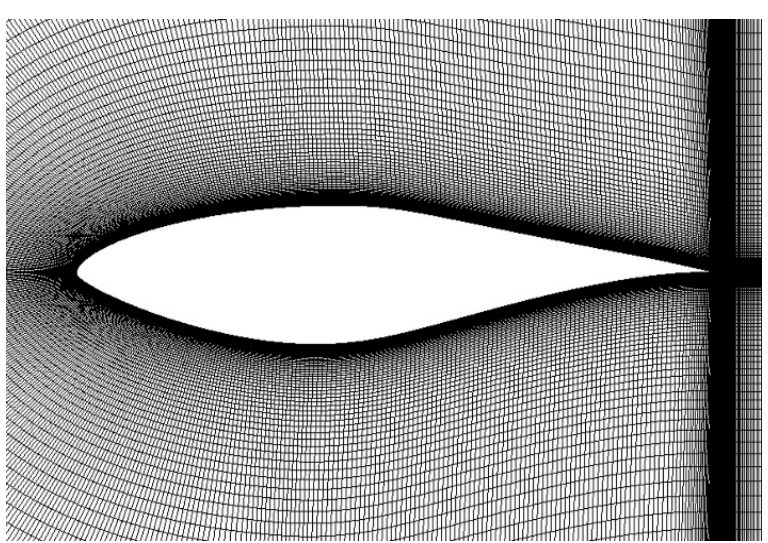

Fig. 6 Structure of the CH-type mesh for the S809 airfoil.

turbulence models show good agreement with the experimental measurements. On the upper side, the location of the transition onset steadily moves toward the leading edge of the airfoil with an accelerated displacement within the range between $5.13^{\circ}$ and $8.2^{\circ}$ on the pressure side, the displacement of the location of the transition onset shows an opposite behavior. With an increasing angle of attack, the transition onset location moves toward the trailing edge of the airfoil, overall showing a significant lower sensitivity regarding a change of the angle of attack. From an angle of approximately $\alpha=8^{\circ}$ on the transition onset location on the upper side remains near the leading edge, offering a significant enlarged surface area appropriated for the riblet application and thus high potential for the viscous drag reduction by the riblet effect. Within this angle range the $k k_{L^{-}} \omega$ model shows a slightly greater consistency with experimental data. On the lower side of the airfoil both models show a 

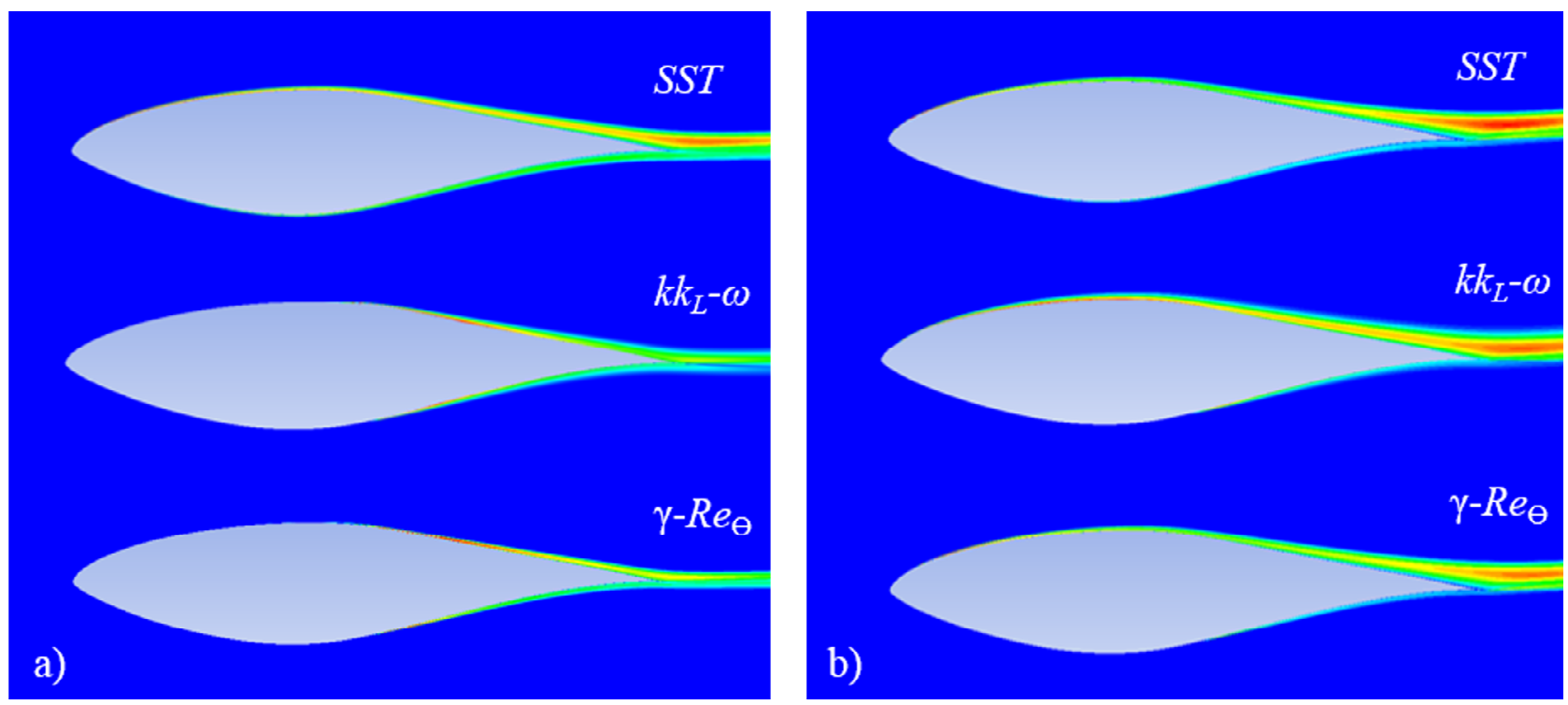

Fig. 7 Contour plots of the turbulent kinetic energy for the S809 airfoil: (a) $\alpha=5.13^{\circ}$ and (b) $\alpha=8.2^{\circ}$.

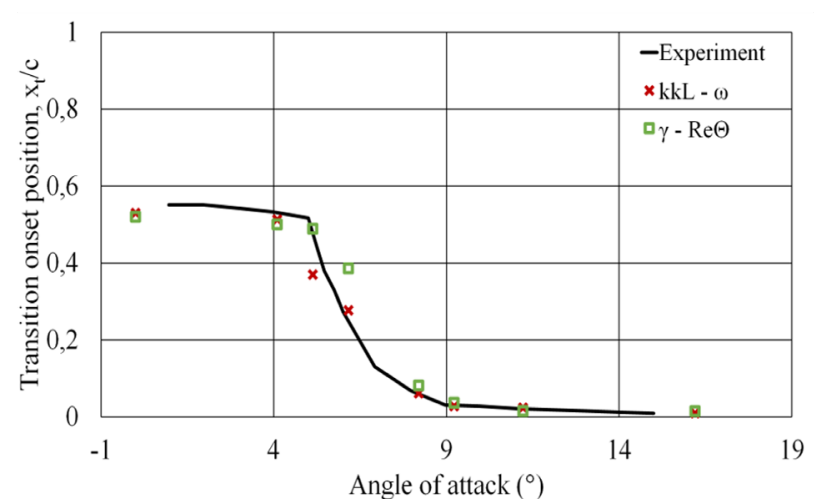

Fig. 8 Predicted location of the transition onset on the upper side of the $\mathrm{S809}$ airfoil.

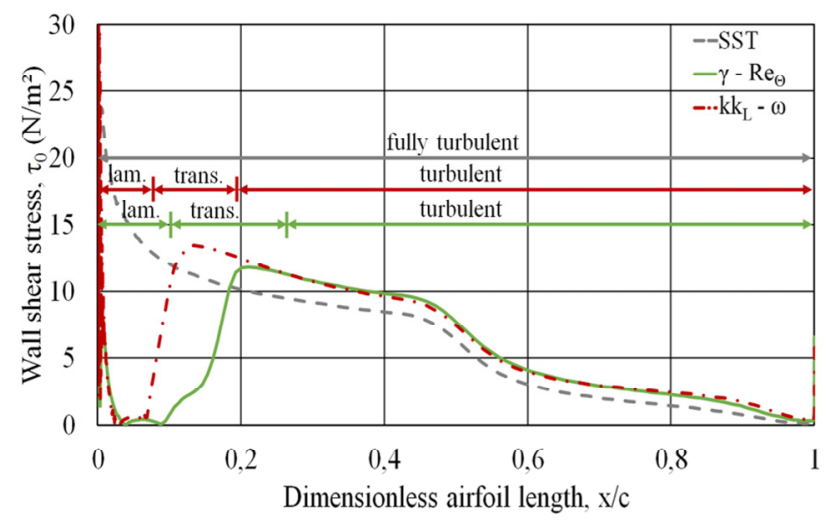

Fig. 10 Computed wall shear stress on the upper side of the S809 airfoil for $\alpha=8.2^{\circ}$.

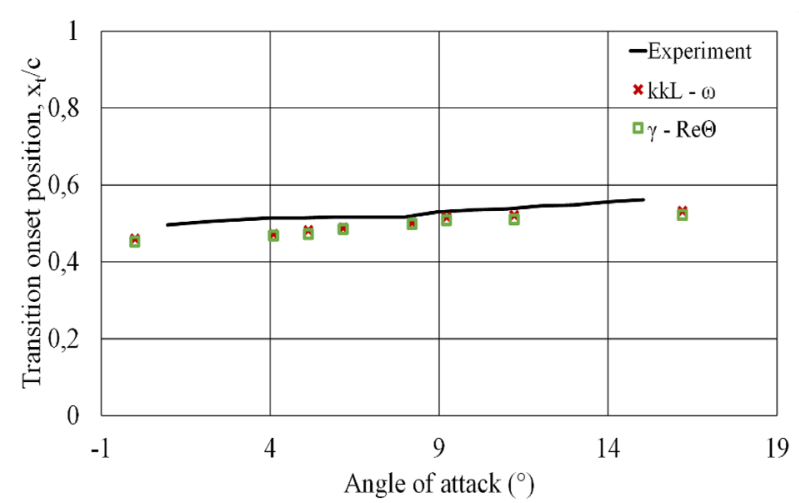

Fig. 9 Predicted location of the transition set on the lower side of the $\mathrm{S809}$ airfoil.

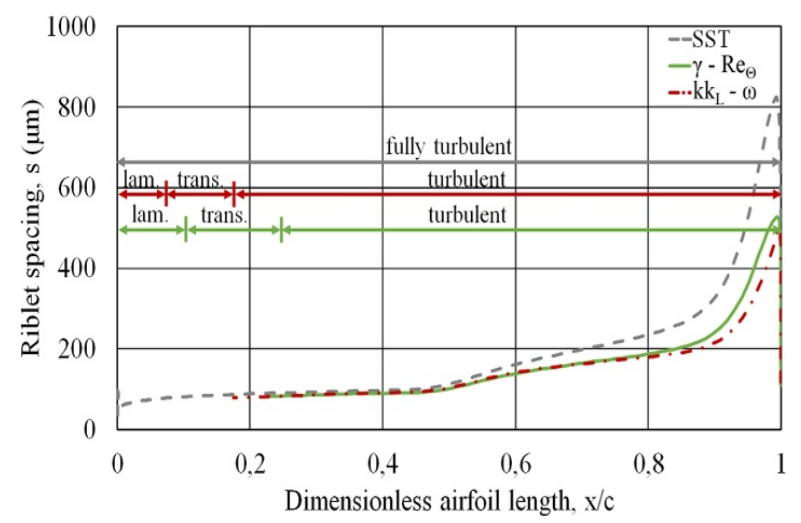

Fig. 11 Computed wall shear stress on the lower side of the $\mathbf{S 8 0 9}$ airfoil for $\alpha=8.2^{\circ}$. 


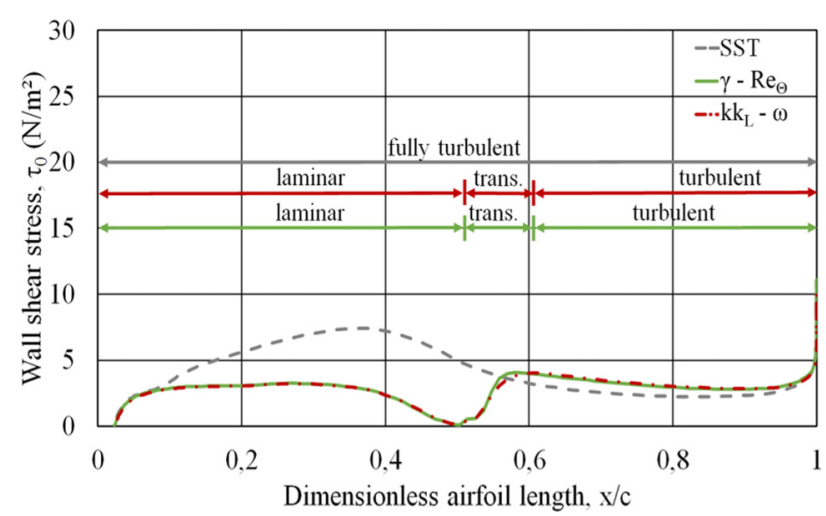

Fig. 12 Computed riblet spacing on the upper side of the S809 airfoil for $\alpha=8.2^{\circ}$.

comparable precision in predicting the transition onset location, whereby in both cases the onset is predicted slightly further upstream in comparison to the experimental data. The determination of the onset location of the fully developed turbulent boundary layer is based on the computed wall shear stress distribution exemplary illustrated in Figs. 10 and 11 for an angle of attack of $\alpha=8.2^{\circ}$. On the lower side of the airfoil both transitional models predict the onset at a dimensionless airfoil length of approximately $x / c=$ 0.6. On the upper side of the airfoil the predicted onset location differs significantly based on the applied model. In case of the $\gamma-\operatorname{Re}_{\Theta}$ model the onset location is predicted at a dimensionless airfoil length of approximately $x / c=0.27$ while the $k k_{L}-\omega$ model predicts the onset location further upstream at an dimensionless airfoil length of $x / c=0.20$, which corresponds to a percentage deviation of the available chord length appropriated for the riblet application in the amount of $9 \%$. Presently it cannot evaluate which model provides more accurate results on the onset location of the fully developed turbulent boundary layer due to unavailable literature data. However, considering the fact, that the application of riblets within the laminar or transitional boundary layer would lead to a local increased drag compared to the smooth surface, at this point the model which predicts the onset location of the fully developed turbulent boundary layer further down-stream should be preferred for the selection of an appropriated area for

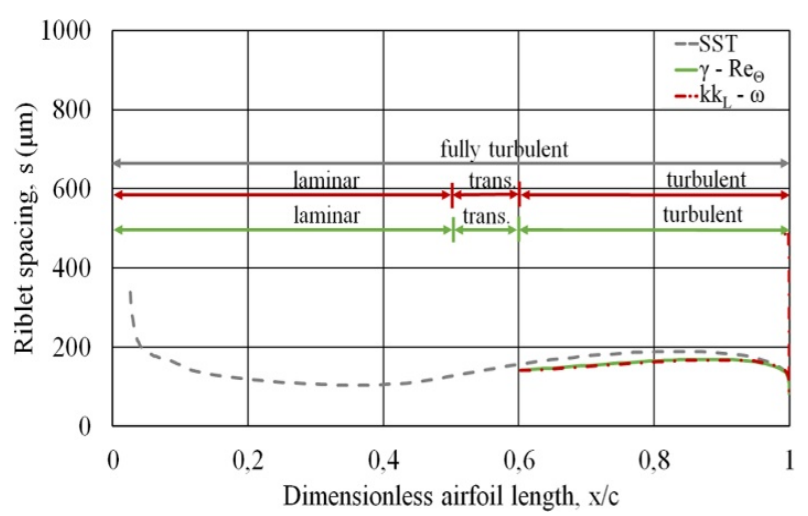

Fig. 13 Computed riblet spacing on the lower side of the S809 airfoil for $\alpha=8.2^{\circ}$.

the riblet application. The computed wall shear stress (cf. Figs. 10 and 11) is also required for the computation of the continuously adapted riblet dimensions. It can be seen that the distributions computed based on the $k k_{L^{-}}-\omega$ and $\gamma-\operatorname{Re}_{\Theta}$ model show a remarkable agreement within the predicted turbulent boundary layer, whereas values computed based on the SST model deviate significantly. The resulting optimal riblet spacing, which can be calculated by rewriting Eqs. (1) and (2):

$$
s=s^{+} v \sqrt{\frac{\rho}{\tau_{0}}}
$$

using a dimensionless riblet spacing of $s^{+}=17$ is illustrated in Figs. 12 and 13. Near the trailing edge on the upper side of the airfoil the computed riblet spacing reaches its maximum of $824 \mu \mathrm{m}, 521 \mu \mathrm{m}$ and $481 \mu \mathrm{m}$ for the SST, $\gamma-\operatorname{Re}_{\Theta}$ and the $k k_{L}-\omega$ model respectively. Referring to the computed riblet spacing based on the SST model this corresponds to a local percentage deviation of $37 \%$ and $42 \%$ for the $\gamma-\operatorname{Re}_{\Theta}$ and $k k_{L^{-}} \omega$ model, whereby a significant effect on the drag reduction can be expected. As reliable literature data for the wall shear stress distribution on the S809 are not available, a statement regarding which turbulence model provides more accurate results on the wall shear stress distribution and is therefore suitable for the computation of continuously adapted riblet dimensions cannot be made. Further investigations are indispensably necessary. 


\section{Conclusion}

In this paper three turbulence models were examined concerning their suitability for the numerical supported design of continuously adapted riblets on the NREL S809 airfoil at various angles of attacks. The $k k_{L^{-}} \omega$ and the $\gamma-\operatorname{Re}_{\Theta}$ model both show a sufficient precise prediction of the transition onset location. In case of high angles of attack, which should be preferred for the riblet application as they provide a significant higher potential for the overall viscous drag reduction, the $k k_{L^{-}} \omega$ model shows a higher consistency with experimental data. In terms of the onset location of the fully developed turbulent boundary layer, the two transitional models differ significantly on the upper side of the airfoil. At this point it could not be finally clarified which model provides more accurate results. However, to avoid a possible drag increase through the application of riblets within the laminar or transitional boundary layer, at this stage the $\gamma-\operatorname{Re}_{\Theta}$ model should be preferred as the onset location of the fully developed turbulent boundary layer is predicted further downstream. Further it has been shown that the $k k_{L}-\omega$ and the $\gamma-\operatorname{Re}_{\Theta}$ models show a remarkable agreement regarding the computed wall shear stress distribution within the predicted fully developed turbulent boundary layer but deviate significant from the computed values using the fully turbulent SST implementation. Therefore, depending on the applied turbulence model the computed riblet dimensions differ locally in the range of up to $42 \%$, whereby a significant impact on the possible drag reduction by the riblet effect can be expected. It is yet not sufficiently investigated, if results based on the transitional models provide more accurate results on the wall shear stress distribution in the turbulent boundary layer compared to a fully turbulent simulation. Presently the authors conduct direct numerical simulations and wind tunnel measurements to provide the data necessary for the final evaluation.

\section{Acknowledgements}

This work has been conducted within the cooperative reach project entitled "Fluidmechanical Optimization of Turbomachinery by High-rate Laser Structuring Technologies (OstrALas)" funded and supported by the German Federal Ministry of Education and Research.

\section{References}

[1] Bechert, D. W., Brusek, M., and Hage, W. 1996. "Drag Reduction with the Slip Wall." AIAA Journal 34 (5): 1072-4.

[2] Siegel, F. 2011. "Abtragen Metallischer Werkstoffe mit Pikosekunden-Laserpulsen für Anwendungen in der Strömungsmechanik.” Dissertation, Hannover, Germany: Gottfried Wilhelm Leibniz Universität Hannover.

[3] Oehlert, K. 2011. "Zur Applikation von Riblets auf Verdichterschaufeln.” Dissertation, München, Germany: Gottfried Wilhelm Leibniz Universität Hannover.

[4] Bechert, D. W., Bartenwerfer, M., Hoppe, G., and Reif, W.-E. 1986. "Drag Reduction Mechanisms Derived from Shark Skin." In 15th Congress of the Intl Council of the Aeronautical Sciences, London, United Kingdom, AIAA: 1044-68.

[5] Cantwell, B., Coles, D., and Dimotakis, P. 1978. "Structure and Entrainment in the Plane of Symmetry of a Turbulent Spot." Journal of Fluid Mechanics 87 (4): 641-72.

[6] Lee, S.-J., and Lee, S.-H. 2001. "Flow Field Analysis of a Turbulent Boundary Layer over a Riblet Surface. Experiments in Fluids." Experiments in Fluids 30 (2): 153-66.

[7] Goldstein, D., Handler, R., and Sirovich, L. 1995. "Direct Numerical Simulation of Turbulent Flow over a Modelled Riblet Covered Surface." Journal of Fluid Mechanics 302: 333-76.

[8] Vukoslavčević, P., Wallace, J. M., and Balint, J. L. 1992. "Viscous Drag Reduction Using Stream-Wise Aligned Riblets." AIAA Journal 30 (4): 1119-22.

[9] Walsh, M. J., Riblets. In Bushnell, D. M., and Hefner, J. N. 1990. Viscous Drag Reduction in Boundary Layers. Washington DC, USA, AIAA.

[10] Bruse, M. 1999. "Zur Strömungsmechanik wandreibungsvermindernder Riblet-Oberflächen.” AIAA Journal 30: 1119-22.

[11] Somers, D. M. 1997. "Design and Experimental Results for the S809 Airfoil." Pennsylvania, U.S.: State College PA, National Renewable Energy Laboratory; January, Technical Report WE711110. 
[12] Menter, F. R. 1994. "Two-Equation Eddy-Viscosity Turbulence Models for Engineering Applications." AIAA-Journal 32 (8): 1598-605.

[13] Aftab, S. M. A., Mohd Rafie, A. S., Razak, N. A., and Ahmad, K. A. 2016. "Turbulence Model Selection for Low Reynolds Number Flows." PLoS ONE 11 (4): e0153755.

[14] Menter, F. R., Langtry, R., and Völke, S. 2006. "Transition Modelling for General Purpose CFD Codes." Flow Turbulence Combust 77 (1-4): 277-303.
[15] Walters, D. K., and Leylek, J. H. 2004. "A New Model for Boundary Layer Transition Using a Single Point RANS Approach.” Journal of Turbomachinery 126 (1): 193-202.

[16] Walters, D. K., and Leylek, J. H. 2003. “A CFD Study of Wake-Induced Transition on a Compressor-Like Flat Plate." Journal of Turbomachinery 6: 565-78.

[17] Eppler, R., and Somers, D. M. 1980. “A Computer Program for the Design and Analysis of Low-Speed Airfoils." NASA; Technical Report NASA-TM-81862. 